\title{
Recurrent Meningitis Secondary to Cervical Neuroenteric Fistula in an Infant with Klippel Feil Syndrome and Esofage Duplication: A Unique Case Report
}

\author{
Soto $\mathrm{C}^{1 *}$, Daoud Z $\mathrm{Z}^{2}$, Llanos $\mathrm{D}^{3}$, Llaneza $\mathrm{A}^{2}$, Gómez Bustamante $\mathrm{G}^{4}$, Ramos $\mathrm{JT}^{2}$ and Aleo $\mathrm{E}^{2}$ \\ ${ }^{1}$ Department of Pediatric Surgery, Hospital Clínico San Carlos, Spain \\ ${ }^{2}$ Department of Pediatric, Hospital Clínico San Carlos, Spain \\ ${ }^{3}$ Department of Radiology, Hospital Clínico San Carlos, Spain \\ ${ }^{4}$ Department of Neurosurgery, Hospital Clínico San Carlos, Spain
}

Submission: January 20, 2020; Published: February 07, 2020

*Corresponding author: Soto C, Department of Pediatric Surgery, Hospital Clínico San Carlos, Madrid, Spain

\author{
Abstract \\ We present a patient with recurrent bacterial meningitis due to different gram-negative bacilli as result of a neuroenteric fistula, oesophageal \\ duplication and Klippel-Feil syndrome. \\ Keywords: Recurrent meningitis; Clostridium hathewayi; Neuroenteric fistula; Oesophageal duplication; Klippel-Feil syndrome
}

\section{Introduction}

Recurrent bacterial meningitis (RBM) in children is an uncommon potentially life-threatening infection that is associated with high rates of morbidity and mortality. The clinical presentation in children is frequently nonspecific, thus diagnosis often gets delayed or missed. RBM is usually associated with a predisposing factor such as congenital defects due to cerebrospinal fluid (CSF) leak or spinal defects, and occasionally secondary to immunodeficiencies, including antibody, complement deficiency or hyposplenism, so the major task, is to identify underlying causes of RBM [1]. The differential diagnoses procedure is based on the age of the patient, the type of cultured microorganism and the associated structural problems. The most common bacterial pathogen causing RBM is Streptococcus pneumonia (50\%), followed by Neisseria meningitidis (25\%), Hemophilus influenzae (6.9\%), Escherichia coli (4.2\%), Proteus spp. (0.9\%) the presence of gram-negative bacilli (GNB), should prompt the search for a neuroentericnteric defect $[1,2]$.

\section{Case Presentation}

A 2 month and 15 days old girl was admitted to the Paediatric Intensive Care Unit (PICU) with fever, lethargy, and poor feeding. She was born after an uncomplicated pregnancy and spontaneous vaginal delivery at $38+5$ weeks with normal birth weight. Vaccination completed for her age including one dose of pneumococcal vaccine. She previously had been discharged from another hospital on day 21, after a 2-week hospitalization to treat a Proteus mirabilis neonatal meningitis, where she received ampicillin and cefotaxime, with complete recovery. On initial assessment, the following features were identified: reduced level of consciousness (response only to painful stimulus); afebrile, normotense, tachycardia (heart rate $190 \mathrm{bpm}$ ); prolonged capillary refill time ( $>5$ seconds peripherally); cold peripheries; tachypnoea (respiratory rate 60) and the arterial oxygen saturation was $96 \%$ with room air. The patient looked ill, irritable and had a full anterior fontanelle. The rest of the examination was 
unremarkable. After fluid resuscitation and a septic workup, the initial empiric therapy included vancomycin and meropenem after a dose of dexamethasone, because of concern for possible sepsis and meningitis. The hemogram and the C-reactive protein (CRP) were normal and Procalcitonin (ProCT) was $6.71 \mathrm{ng} / \mathrm{ml}$ (normal values $<0,5 \mathrm{ng} / \mathrm{ml}$ ). CSF was turbid and showed $740 \mathrm{WBC} / \mathrm{mm} 3$ with $70 \%$ neutrophils, $30 \%$ monocytes. CSF protein was $156 \mathrm{mg} /$ $\mathrm{dl}$, glucose $35 \mathrm{mg} / \mathrm{dl}$ (simultaneous blood glucose $80 \mathrm{mg} / \mathrm{dl}$ ) and gram strain did not reveal any findings. Blood culture was sterile.
Culture of her CSF grew Escherichia coli susceptible to cefotaxime and amoxicillin-clavulanic acid. After this result became available, vancomycin was suspended. A brain MRI (T1 flair signal) was performed and revealed several areas of leptomeningeal highlight probably attributable to meningitis. It was also performed a barium enema that was normal and a radionuclide cisternogram was negative for CSF leak or sinus tracts via nasopharynx and auditory canals. The results of tests for HIV infection and immunodeficiency workup were negative.

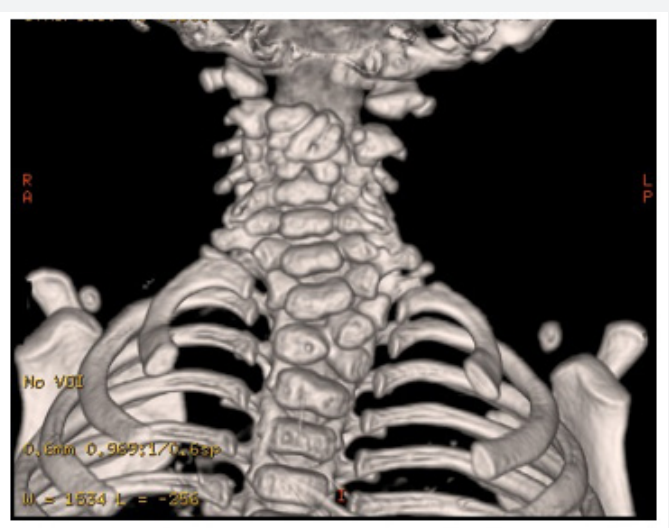

Figure 1: Klippel-Feil anomaly.

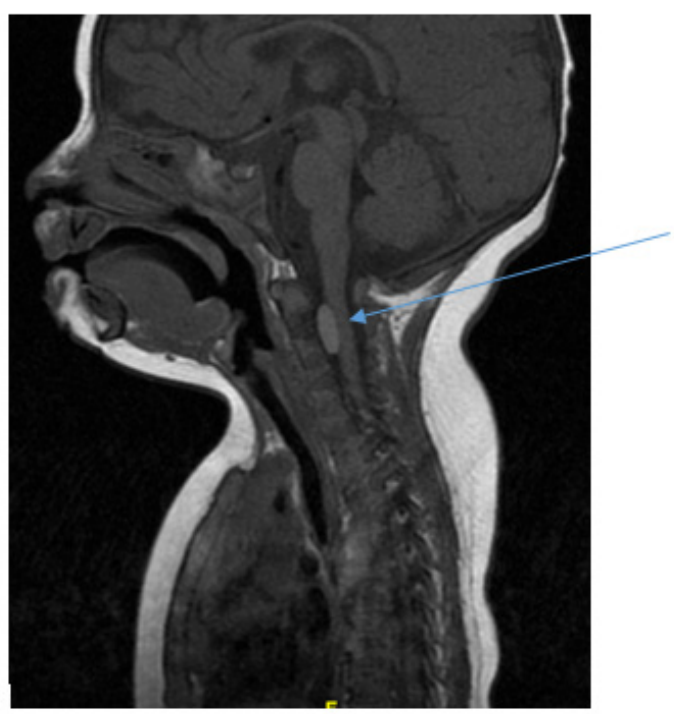

Cyst

Figure 2: Brain and spinal cord MRI with contrast.

The patient was discharged after a complete treatment of 21 days of meropenem and two control CSF sterile with complete recovery. At 4 months of age, one week after discharge, she was readmitted to the PICU with fever, tachycardia, tachypnoea, lethargic, hypotonic and poor feeding. Laboratory studies showed a normal total white blood cell count with mild predominance of neutrophils without any immature forms. She had anaemia with $8,3 \mathrm{~g} / \mathrm{dL}$, haematocrit $25.9 \%$, mean corpuscular volume (MCV) 78.2fL; platelets were 206.000 and CRP was $14.2 \mathrm{mg} / \mathrm{dl}$. Blood, CSF, urine and stool samples were collected for bacterial and virological study. CSF was turbid and revealed a leukocyte count of $1.440 \mathrm{WBC} / \mathrm{mm}^{3}$ with $70 \%$ neutrophils, glucose level of $8 \mathrm{mg} /$ 
dl (the serum glucose level was $90 \mathrm{mg} / \mathrm{dl}$ ). Gram's staining of a sample of her CSF showed several gram-negative, rod-shaped bacteria. Empirical antimicrobial therapy was started again with meropenem. The microbiology laboratory informed 24 hours later about the growth in CSF of gram-positive cocci in clusters, and vancomycin was added. Clostridium hathewayi susceptible to meropenem, clindamycin, amoxicillin-clavulanic acid and metronidazole grew in blood culture. In addition, in her CSF grew a Staphylococcus epidermidis. Brain and spinal cord MRI evidenced a cervical hemivertebrae fusion from C2 to C5 in the context of a Klippel-Feil anomaly (Figure 1); the presence of a
$1.4 \mathrm{~cm}$ cystic intradural and extramedular mass and a fistulous lesion with a $2.5 \mathrm{~cm}$ craniocaudal diameter, communicating the C3-vertebral body with the left retropharyngeal space (Figure 2 \& 3 ). The esophagogastrogram confirmed the presence of a tubular mass connecting the retropharyngeal space with the oesophagus (Figure 4). With the diagnosis of neuroenteric cyst, esophagus duplication and Klippel-Feil syndrome, the patient was dismissed with prophylactic treatment with ceftibuten until the surgery was scheduled. However, she was re-hospitalized at 7 months of age, with a history of irritability and poor feeding during 5 days, without fever or other symptoms.
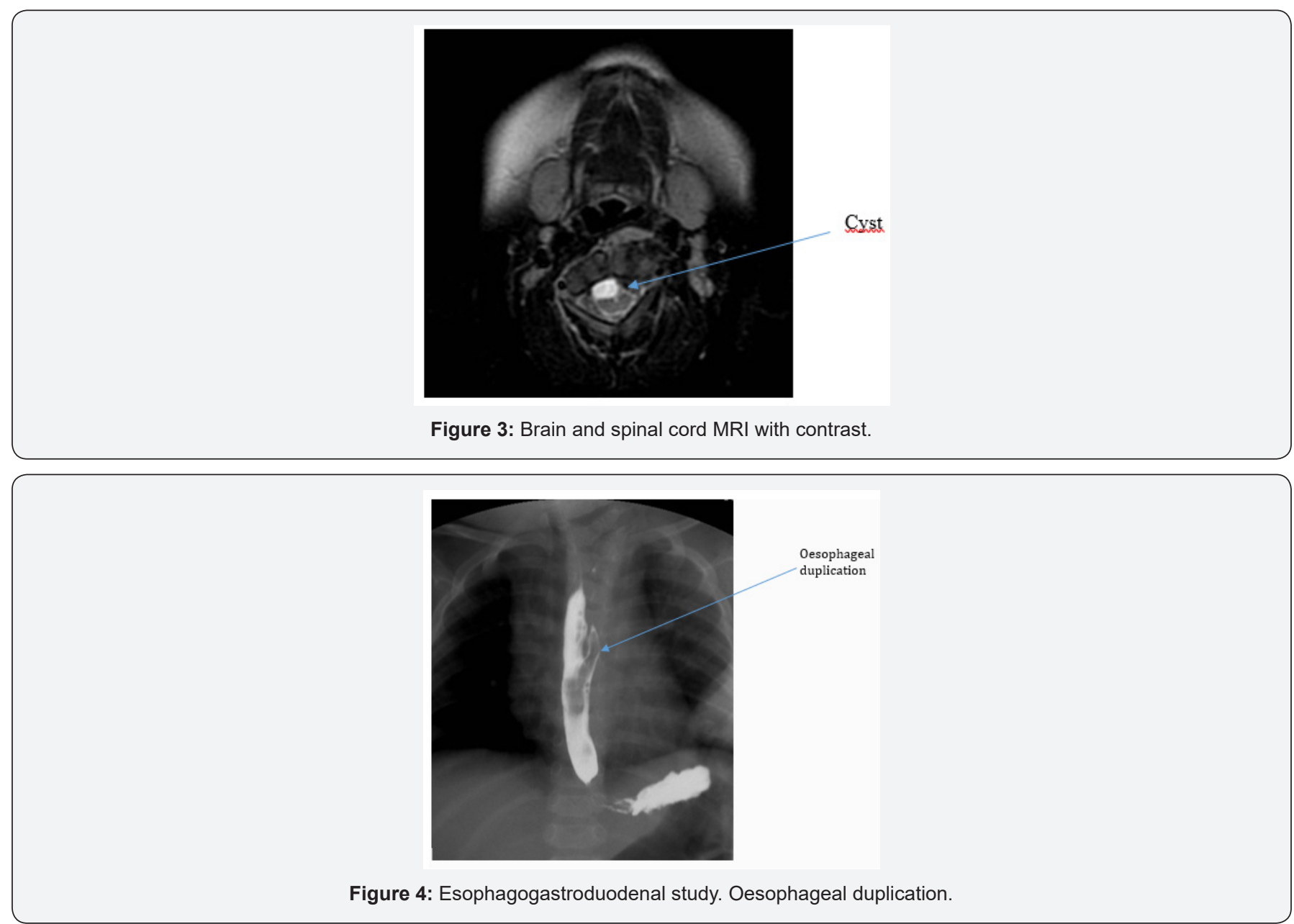

Physical examination revealed an irritable but alert infant with a normal anterior fontanel, hypotonic and somnolent; she was febrile with an axillary temperature of $38.6^{\circ} \mathrm{C}$, poorly perfused, respiratory rate of 45 breaths per minute and tachycardia (192bpm), and with normal oxygen saturation. Laboratory testing performed at admission revealed a peripheral WBC count of 14.90 cells $/ \mathrm{mL}$, with $67.2 \%$ segmented neutrophils, $25.2 \%$ lymphocytes and $4 \%$ monocytes. Measurement of hemoglobin and platelet count, serum electrolytes, creatinine, creatine phosphokinase, and transaminase values were within normal. CRP $1.53 \mathrm{mg} / \mathrm{dl}$ and Procalcitonin $6.71 \mathrm{ng} / \mathrm{ml}$. Lumbar puncture revealed a turbid CSF with leukocyte count of $8000 \mathrm{WBC} / \mathrm{mm}^{3}$ with absolute neutrophils (98\%), no detectable glucose and a protein level of $448 \mathrm{mg} / \mathrm{dl}$. Gram's staining of a sample of her CSF showed several gram-negative bacilli and empiric treatment with meropenem was started. In blood and cerebrospinal fluid grew an Acinetobacter genospecies susceptible to tigecyclin, imipenem, piperacillin-tazobactam, gentamycin, and amoxicillin-clavulanic acid. Two weeks later, the child underwent a surgical procedure in collaboration by neuro and paediatric surgery teams. The esophageal duplication was removed through a thoracic approach. The retropharyngeal lesion through a right cervicectomy although 
the intradural cystic mass was resected through a laminectomy of the C3 vertebral body. The bone defect was repaired with protesic material to avoid leakage of CSF. Histological examination of the excised tissue showed a cyst covered by ciliated cuboidal cells, similar to respiratory tissue, confirming the diagnosis of Neuroenteric cyst. However, a non-symptomatic CSF collection developed ventrally to the C3-vertebral body. Conservative treatment failed and the collection was finally removed, and CSF leakage closed. Six years after surgery the patient is doing well, asymptomatic without any neurological sequelae and no other episodes of meningitis.

\section{Discussion}

RBM is uncommon in children. It has been estimated that $1.3 \%$ of all of bacterial meningitis develop recurrences [3]. A recurrence is defined as a new episode occurring at least 3 weeks after CSF sterilization. It may be due to the same or a different microorganism. The type of bacteria isolated can lead to suspicion of the predisposing condition or the possible underlying deficiency. Tebruegge et al. [2] described that 59\% of RBM were related to anatomical problems, $36 \%$ to immunodeficiencies, and $5 \%$ to chronic Para meningeal infections. The $48 \%$ of the anatomical problems were cases of traumatic head injury with secondary CSF fistula, followed by inner ear abnormalities (26\%) and Neuroenteric cyst (9\%). Neuroenteric cyst (NE) is a rare congenital abnormality that develops during the third week of embryonic life, is a foregut duplication cyst that results from failure of separation of ectoderm from the endoderm. It has a variety of clinical presentations depending on size and location $[4,5]$. There are two major histologic patterns suggesting respiratory or gastrointestinal tract origins. The histology in our patient suggested a respiratory tract origin of the cyst. These cysts generally occur in the spine, like our patient and rarely occur intracranially [6]. Although NE cysts are benign lesions, the natural history of untreated intraspinal NE cysts is unfavorable. The proper treatment of NE cysts is complete surgical removal [7]. Congenital vertebral anomalies are frequently seen in patients with $\mathrm{NE}$, suggesting a common error in embryological development. Klippel-Feil syndrome is a congenital spinal malformation characterized by the failure in segmentation of two or more vertebrae, primarily affecting the cervical spine, accompanied by a spectrum of neurologic anomalies [8]. Vertebral body anomalies, as seen in Klippel-Feil syndrome can be found anywhere from the cervical to the sacral region, indicating a connection between the neural canal and the gastrointestinal system [9]. This congenital anomaly likely results when the embryonic connection between notochord and endoderm fails to reabsorb. Gray et al. [10] describe an association of NE, fistulae and gastrointestinal duplications in $5 \%$ of the Klippel-Feil patients.

Our patient had four episodes of bacterial meningitis with different microorganism. This case is unique because of the anatomical defect and also because of the etiology due to Clostridium hathewayi, in our best knowledge this is the first report of disease caused by this microorganism in children. C. hathewayi was first reported in 2001 by Steer et al. [11] is an anaerobic, endospore-forming, gram-negative stain rodshaped bacteria with subterminal, oval-to-round endospores. Its growth in blood cultures has been reported in a few cases in the literature. Elsayed et al. In 2004 described a 27-year-old man with acute cholecystitis, hepatic abscess, and bacteremia [12]; Woo et al. in 2004 causing bacteremia in a 39 old patient with acute appendicitis [13]; Linscott et al. in 2005 reported a septicemia due to Clostridium hathewayi and Campylobacter hominis [14]. Dababneh et al. in 2014 bacteraemia and surgical site infection after uterine myomectomy in a 42-year-old woman [15], Tena et al. in 2014 a Fournier's gangrene in 73-year-old man [16] and Randazzo in 2015 an acute appendicitis in 60-year-old man [17]. It is possible that Clostridium hathewayi only grew in blood culture and not in CSF because of the special needs for its grow and the isolation of $S$. epidermidis as well. We think that $C$. hathewayi was a time pathogen and $S$. epidermidis would be likely contaminated due to the high cell counts in CSF. In summary, the development of RBM due to a variety of microorganism should prompt the investigation of an anatomical defect, particularly a neuroenteric fistula when GNB are involved.

\section{Conflicts of interest}

The authors declare that there is no conflict of interests regarding the publication of this paper.

\section{Acknowledgement}

Celia Gil L. MD PhD and Belén Joyanes A. MD PhD from department of Pediatric and Arribi A, MD department of Microbiology of Clínico San Carlos Hospital. Madrid. Spain.

\section{References}

1. Livingston R, Harrison C (2012) Recurrent Meningitis. Principles and Practice of Pediatric Infectious Diseases. Long S $4^{\text {th }}$ Edn. Elsevier.

2. Tebruegge M, Curtis N (2008) Epidemiology, etiology, pathogenesis, and diagnosis of recurrent bacterial meningitis. Clin Microbiol Rev 21(3): 519-537.

3. Drummond DS, de Jong AL, Giannoni C, Sulek M, Friedman EM (1999) Recurrent meningitis in the pediatric patient. International Journal of Pediatric Otorhinolaryngology 48(3): 199-208.

4. Giles W Becker, Robert De Battersby (2004) Spinal neuroentericnteric cyst presenting as recurrent midline sebaceous cysts. Ann R Coll Surg Engl 87(1): 1-4.

5. Esfahani DR, Burokas L, Brown HG, Hahn YS, Nikas D (2017) Management of an unusual, recurrent neuroentericnteric cyst in an infant: case report and review of the literature. Childs Nerv Syst 33(9): 1603-1607.

6. Gill Kiran DO, Zachary Bryan MSIV, Williams Candice, Raffel Corey, Boue Daniel, et al. (2010) Recurrent Meningitis in a Teenager. The Pediatric Infectious Disease Journal 29(10): 984, 988-989. 
7. Anil Can, Ellianne J Dos Santos Rubio, BasJasperse, Robert M Verdijk, B SanjayHarhangi (2015) Spinal Neuroentericnteric Cyst in Association with Klippel-Feil Syndrome: Case Report and Literature Review. World Neurosurg 84(2): 592, 599-614.

8. Gumerlock Mary Kay, Spollen Linda E, Nelson Martin J (1991) Cervical neuroentericnteric fistula causing recurrent meningitis in Klippel-Feil sequence: case report and literature review. Pediatr Infect Dis J 10(7): 532-535.

9. Alrabeeah A, Gillis DA, Giacomantonio M, Lau H (1988) Neuroentericnteric cysts-a spectrum. J Pediatr Surg 23(8): 752-754.

10. Gray SW, Romaine CB, Skandalakis JE (1964) Congenital Fusion of the Cervical Vertebrae. Surg Gynecol Obstet 118: 373-385.

11. Steer T Collins, Gibson GR, Hippe H, Lawson PA (2001) Clostridium hathewayi from human faeces. Syst Appl Microbiol 24(3): 353-357.

12. Elsayed S, Zhang K (2004) Human infection caused by Clostridium hathewayi. Emerg Infect Dis 10(11): 1950-1952.
13. Patrick CY Woo, Susanna KP Lau, Gibson KS Woo, Ami MY Fung, Viola PY Yiu, et al. (2004) Bacteremia Due to Clostridium hathewayi in a Patient with Acute Appendicitis. J Clin Microbiol 42(12): 5947-5949.

14. Linscott AJ, Flamholtz RB, Shukla D, Song Y, Liu C, et al. (2005) Fatal septicemia due to Clostridium hathewayi and Campylobacter hominis. Anaerobe 11(1-2): 97-98.

15. Dababneh AS, Nagpal A, Palraj BR, Sohail MR (2014) Clostridium hathewayi bacteraemia and surgical site infection after uterine myomectomy. BMJ Case Rep 4: 2014

16. Tena D, Losa C, Medina-Pascual MJ, Sáez-Nieto JA (2014) Fournier's gangrene caused by Actinomyces funkei, Fusobacterium gonidiaformans and Clostridium hathewayi. Anaerobe 27: 14-16.

17. Adrien Randazzo, Anne Kornreich, Bénédicte Lissoir (2015) A Clostridium hathewayi isolate in blood culture of a patient with an acute appendicitis. Anaerobe 35: 44-47. 